\title{
Extended Antitumor Response of a BRAF V600E Papillary Thyroid Carcinoma to Vemurafenib
}

\author{
Siraj M. Ali ${ }^{a}$ Je He ${ }^{a}$ Wade Carson ${ }^{b}$ Phil J. Stephens ${ }^{a}$ Joseph Fiorillo ${ }^{b}$ \\ Doron Lipson $^{\mathrm{a}}$ Gary A. Palmer ${ }^{\mathrm{a}}$ Jeffrey S. Ross ${ }^{\mathrm{a}}$ Vincent A. Miller ${ }^{\mathrm{a}}$ \\ Jeffrey Sharman ${ }^{\text {b }}$ \\ ${ }^{a}$ Foundation Medicine, Inc., Cambridge, Mass., and ${ }^{b}$ Willamette Valley Cancer Institute, \\ Eugene, Oreg., USA
}

\section{Key Words}

Papillary thyroid carcinoma $\cdot$ BRAF V600E $\cdot$ Vemurafenib

\begin{abstract}
Context: For patients with metastatic papillary thyroid carcinoma (PTC) refractory to radioactive iodine (RAI) treatment, systemic chemotherapy has limited efficacy. Such tumors frequently harbor $B R A F \mathrm{~V} 600 \mathrm{E}$, and this alteration may predict responsiveness to vemurafenib treatment. Objective: We report a metastatic PTC patient refractory to RAI treatment that underwent genomic profiling by next-generation sequencing. The sole genomic alteration identified was BRAF V600E on a near diploid genome with trisomy 1q. With vemurafenib treatment, the patient experienced a dramatic radiographic and clinical improvement, with the duration of an ongoing antitumor response exceeding 23 months. Design: Hybridization capture of 3,769 exons of 236 cancer-related genes and the introns of 19 genes frequently rearranged in cancer was applied to $>50 \mathrm{ng}$ of DNA extracted from a formalin-fixed, paraffin-embedded biopsy of a lymph node containing metastatic PTC and was sequenced to a high, uniform coverage of $\times 616$. Results: A BRAF V600E alteration was identified with no other somatic genomic alterations present within a near diploid tumor genome. The patient initially received vemurafenib at $960 \mathrm{mg}$ twice daily that was reduced to $480 \mathrm{mg}$ twice daily due to rash and diarrhea and has experienced an ongoing antitumor response exceeding 23 months by both PET-CT and dedicated CT imaging. Conclusions: Genomic profiling in metastatic, RAI-refractory PTC can reveal a targetable BRAF V600E alteration without compounding somatic alterations, and such patients may derive a more
\end{abstract}


prolonged benefit from vemurafenib treatment. Prospective clinical trials are ongoing to confirm our preliminary observation.

(c) 2014 S. Karger AG, Basel

\section{Introduction}

Papillary thyroid carcinoma (PTC) is the most common (80-85\%) form of thyroid carcinoma and carries an excellent prognosis with the 25-year survival exceeding 95\% $[1,2]$. However, a subset of PTC patients will develop recurrent metastatic disease, with estimates of this population ranging from 15 to $30 \%[3,4]$. Half of such patients fail radioactive iodine (RAI), and systemic chemotherapy has minimal benefit in this clinical situation [3]. Multikinase small molecule inhibitors such as sorafenib and vandetanib can be used per recent NCCN guidelines (Version 2.2013), but prospective clinical trials have not yet demonstrated a distinct molecular subgroup of PTC patients that derives large benefit from such targeted therapies [5].

$B R A F$ V600E is present in approximately $45 \%$ of all PTC [6], with alterations of CTNNB1, HRAS, IDH1, KRAS, NRAS, PIK3CA and TP53 being less common. BRAF V600E is found more frequently in the classic and tall cell histologic variants [7] and is under investigation as a biomarker for diagnosis, prognosis and targeted treatment $[8,9]$. For the latter, clinical trials are underway with vemurafenib as a treatment for metastatic PTC with BRAF V600E, and preliminary results are promising [10]. We report here a patient harboring $B R A F$ V600E in her PTC as the sole somatic driver alteration identified by comprehensive genomic profiling by next-generation sequencing and with a durable antitumor response to vemurafenib.

\section{Materials and Methods}

Tissue from an incisional lymph node biopsy was submitted as a formalin-fixed, paraffin-embedded block to a CLIA-certified, CAP-accredited laboratory (Foundation Medicine, Cambridge, Mass., USA). The lymph node was selected for submission as this was the most recent and surgically accessible recurrent metastatic lesion. Hybridization capture of 3,769 exons from 236 cancer-related genes and 47 introns of 19 genes frequently rearranged in cancer was applied to $>50$ ng of DNA extracted from the block and sequenced to a median coverage of $\times 616$ [11]. For thyroglobulin and antithyroglobulin testing, chemiluminescent immunoassays were performed.

\section{Results}

In 2006, a 73-year-old woman with a prior history of early-stage breast cancer treated with lumpectomy, radiation and adjuvant tamoxifen presented with a neck mass at routine follow-up. Ultrasound evaluation of the neck showed a solid isoechoic mass contiguous with an enlarged left thyroid lobe with resulting right tracheal deviation. A CT scan showed four discrete neck masses, three on the left and one on the right. The primary nodule arising from the thyroid measured 4 by $3.2 \mathrm{~cm}$ and abutted the esophagus and the carotid sheath. The other lesions were felt most consistent with nodal metastases.

A fine-needle aspiration biopsy demonstrated a cytology characteristic of a papillary thyroid lesion. A thyroidectomy with bilateral modified radical neck dissection was performed, and pathologic examination identified a well-differentiated PTC, which measured 
Ali et al.: Extended Antitumor Response of a BRAF V600E Papillary Thyroid Carcinoma to Vemurafenib

$4.0 \mathrm{~cm}$ in greatest dimension (fig. 1a). Extrathyroidal extension and invasion of skeletal muscle and fat was present as well as multiple fused ipsilateral lymph nodes and contralateral nodes. The patient's disease was staged as T4N1bM0, stage IVa. I ${ }^{131}$ imaging revealed focal intense radioiodine uptake within the thyroid bed. The patient was treated with 200 $\mathrm{mCi}$ of $\mathrm{I}^{131}$ and placed on thyroid-stimulating hormone suppression.

In February 2012, the patient presented with progressive fatigue, dyspnea and neck fullness. A CT scan demonstrated innumerable pulmonary nodules, extensive mediastinal and hilar lymphadenopathy. A core needle biopsy of the lymph nodes demonstrated recurrence of well-differentiated PTC; dedifferentiation was not observed. Positron emission tomography (PET) imaging showed ${ }^{18} \mathrm{~F}$-fluorodeoxyglucose-avid disease that extensively involved the base of the neck, mediastinum, pulmonary parenchyma and the pleura (fig. 2a).

$I^{131}$ imaging showed residual uptake in the neck and diffuse disease within the lungs, cervical and mediastinal lymph nodes. A second course of $\mathrm{I}^{131}$ was administered, and PET imaging showed some regression of her neck lesion associated with mild improvement in respiratory symptoms, but the remainder of disease was unaffected (fig. 2b). Due to persistent dyspnea, additional therapeutic options were pursued. The patient's PTC underwent a comprehensive genomic profile and was identified as harboring BRAF V600E.

$B R A F$ V600E was the sole genomic alteration identified in this specimen (fig. 1b), which did not contain tall cell features. This alteration occurred at a mutant allele frequency of $34 \%$ in the background of an estimated tumor content of $30 \%$, as determined histologically. No focal high-level amplifications, small somatic insertions/deletions or rearrangements were present, and the tumor genome was estimated to be near diploid with trisomy 1q.

Risks and benefits were discussed, and the patient provided informed consent to proceed with vermurafenib treatment. She started with $960 \mathrm{mg}$ of vemurafenib twice daily but experienced an extensive erythematous, pruritic rash. The treatment was briefly withheld and then resumed at $480 \mathrm{mg}$ twice daily. The subsequent therapy was notable for a mild increase in bowel frequency; however, a significant improvement in dyspnea was observed. Two months later, repeat PET imaging demonstrated a dramatic reduction in both ${ }^{18} \mathrm{~F}$ fluorodeoxyglucose avidity and mass size (fig. 2 c) quantified as $76 \%$ by RECIST criteria and equally distributed over the pulmonary and nodal tissue. At 6 months, the patient continued to tolerate vemurafenib well, and subsequent imaging showed further improvement in disease burden (fig. 2d). In parallel, the patient's antithyroglobulin measurements decreased, although thyroglobulin was less informative and likely secondary to the high antithyroglobulin titer, which can interfere with assaying thyroglobulin levels (table 1). She remains on therapy at 23 months and the disease is well controlled with few remaining symptoms attributable to her malignancy.

\section{Discussion}

The mortality associated with PTC is concentrated in a subset of patients who develops recurrent, metastatic disease [2]. Treatment for such patients is dependent on the success of RAI, as systemic cytotoxic therapy is minimally effective. The substantial prevalence of $B R A F$ V600E in PTC suggests potential responsiveness to treatment with vemurafenib, a small molecular kinase inhibitor known to be highly specific for BRAF V600E [12].

Clinical trials have demonstrated a subset of melanoma patients harboring BRAF V600E who respond well to vemurafenib treatment, leading to FDA approval of the drug. In contrast, patients with colon carcinoma containing the same alteration typically do not benefit from vemurafenib treatment [13]. The differential response between tumor types 
Ali et al.: Extended Antitumor Response of a BRAF V600E Papillary Thyroid Carcinoma to Vemurafenib

harboring BRAF V600E necessitates tumor-specific clinical investigations for assessing responsiveness to treatment with vemurafenib.

A recent phase I study of vemurafenib in patients with metastatic solid tumors reported responses from 3 patients with PTC harboring BRAF V600E [10]. After receiving $729 \mathrm{mg}$ of vemurafenib on protocol, a retrospective molecular analysis of archived tumor tissue from these patients identified BRAF V600E in all of these samples, which is consistent with BRAF V600E present in $80 \%$ of the metastatic PTC patients. Two patients had stable disease, and the remaining patient had a partial response, as assessed by RECIST, with an average time to progression of 12.1 months [10]. Similarly, a phase II trial [14] treated 25 targeted therapynaïve PTC patients with $B R A F \mathrm{~V} 600 \mathrm{E}$ with vemurafenib. Thirty-five percent of these patients had a partial response, and the mean progression-free survival was 15.6 months.

The targeted therapy-naïve patient described here received prospective genomic profiling that identified $B R A F \mathrm{~V} 600 \mathrm{E}$ as the only genomic alteration in a near diploid background. The lack of other driver alterations potentially suggests a strong reliance of tumor proliferation on BRAF V600E, a hypothesis that is supported by both the magnitude and duration ( $>23$ months and ongoing) of antitumor response to vemurafenib. This patient also did not experience squamous dysplasia on vemurafenib, in contrast to 2 of 3 patients in the phase I study [10]. It remains to be clarified whether both toxicity and the duration of the antitumor response can be linked to vemurafenib dosage, and whether the efficacy of the dosage is in turn dependent on the genomic profile of the tumor.

The exceptional antitumor response of this PTC patient with BRAF V600E suggests that a subset of patients may derive greater benefit from vemurafenib treatment, potentially with similar genomic profiles as the patient described herein. This therapeutic hypothesis should be investigated as clinicians caring for patients with metastatic RAI-refractory disease may consider utilizing genomic profiling.

\section{Disclosure Statement}

S.M.A., J.H., P.J.S., D.L., G.A.P., J.S.R. and V.A.M. are employed by and have equity interest in Foundation Medicine, Inc. W.C., J.F. and J.S. have nothing to declare.

\section{References}

1 Mazzaferri EL, Kloos RT: Clinical review 128: current approaches to primary therapy for papillary and follicular thyroid cancer. J Clin Endocrinol Metab 2001;86:1447-1463.

2 McLeod DS, Sawka AM, Cooper DS: Controversies in primary treatment of low-risk papillary thyroid cancer. Lancet 2013;381:1046-1057.

3 Busaidy NL, Cabanillas ME: Differentiated thyroid cancer: management of patients with radioiodine nonresponsive disease. J Thyroid Res 2012;2012:618985.

-4 Mazzaferri EL, Jhiang SM: Long-term impact of initial surgical and medical therapy on papillary and follicular thyroid cancer. Am J Med 1994;97:418-428.

5 Xing M, Haugen BR, Schlumberger M: Progress in molecular-based management of differentiated thyroid cancer. Lancet 2013;381:1058-1069.

-6 Xing M: BRAF mutation in papillary thyroid cancer: pathogenic role, molecular bases, and clinical implications. Endocr Rev 2007;28:742-762.

7 Mazzaferri EL: Histologic variants of papillary thyroid carcinoma. Endocr Pract 2001;7:139-142.

8 Mazzaferri E: Fine-needle aspiration biopsy, ultrasound and BRAF(v600E) analysis - is this the best FNAB combination for most patients? Clin Endocrinol (Oxf) 2011;75:740-742.

-9 Xing M, Alzahrani AS, Carson KA, Viola D, Elisei R, Bendlova B, Yip L, Mian C, Vianello F, et al: Association between BRAF V600E mutation and mortality in patients with papillary thyroid cancer. JAMA 2013;309:1493-1501. 


\section{Case Reports in Oncology}

\begin{tabular}{l|l}
\hline Case Rep Oncol 2014;7:343-348 \\
\hline DOI: $10.1159 / 000363377$ & $\begin{array}{l}\text { C 2014 S. Karger AG, Basel } \\
\text { www.karger.com/cro }\end{array}$ \\
\hline
\end{tabular}

Ali et al:: Extended Antitumor Response of a BRAF V600E Papillary Thyroid Carcinoma to Vemurafenib

10 Kim KB, Cabanillas ME, Lazar AJ, Williams MD, Sanders DL, Ilagan JL, Nolop K, Lee RJ, Sherman SI: Clinical responses to vemurafenib in patients with metastatic papillary thyroid cancer harboring BRAF mutation. Thyroid 2013;10:1277-1283.

11 Frampton GM, Fichtenholtz A, Otto GA, Wang K, Downing SR, He J, Schnall-Levin M, White J, Sanford EM, et al: Development and validation of a clinical cancer genomic profiling test based on massively parallel DNA sequencing. Nat Biotechnol 2013;31:1023-1031.

12 Bollag G, Tsai J, Zhang J, Zhang C, Ibrahim P, Nolop K, Hirth P: Vemurafenib: the first drug approved for BRAF-mutant cancer. Nat Rev Drug Discov 2012;11:873-886.

13 Prahallad A, Sun C, Huang S, Di Nicolantonio F, Salazar R, Zecchin D, Beijersbergen RL, Bardelli A, Bernards R: Unresponsiveness of colon cancer to BRAF(V600E) inhibition through feedback activation of EGFR. Nature 2012;483:100-103.

14 Brose MS, Cabanillas ME, Cohen EEW, et al: An open-label, multi-center phase 2 study of the BRAF inhibitor vemurafenib in patients with metastatic or unresectable papillary thyroid cancer (PTC) positive for the BRAF V600 mutation and resistant to radioactive iodine (abstract LBA28). Presented at the European Cancer Congress 2013, Amsterdam, September 27-October 1, 2013.

Table 1. Clinical events, thyroglobulin and antithyroglobulin levels

\begin{tabular}{llcc}
\hline Date & Event & Thyroglobulin & $\begin{array}{c}\text { Antithyroglob- } \\
\text { ulin (Ab) }\end{array}$ \\
& & 3.72 & 4,370 \\
$6 / 9 / 2011$ & & 8.17 & 10,072 \\
$4 / 2 / 2012$ & & \\
$4 / 9 / 2012$ & Second course of RAI & \\
$5 / 8 / 2012$ & PET scan; limited & \\
& response & & \\
$6 / 11 / 2012$ & Vemurafenib initiated & \\
$1 / 21 / 2013$ & & 5.28 & 1,962 \\
$2 / 18 / 2013$ & & 7.04 & 1,802 \\
$6 / 18 / 2013$ & & 3.21 & 820 \\
$9 / 12 / 2013$ & & 4.96 & 531 \\
\hline
\end{tabular}

Chemiluminescent immunoassays were performed. Please note that thyroglobulin values could not be interpreted secondary to antibody interference. 


\section{Case Reports in Oncology}

Case Rep Oncol 2014;7:343-348

DOI: 10.1159/000363377

2014 S. Karger

www.karger.com/cro

Ali et al.: Extended Antitumor Response of a BRAF V600E Papillary Thyroid Carcinoma to Vemurafenib

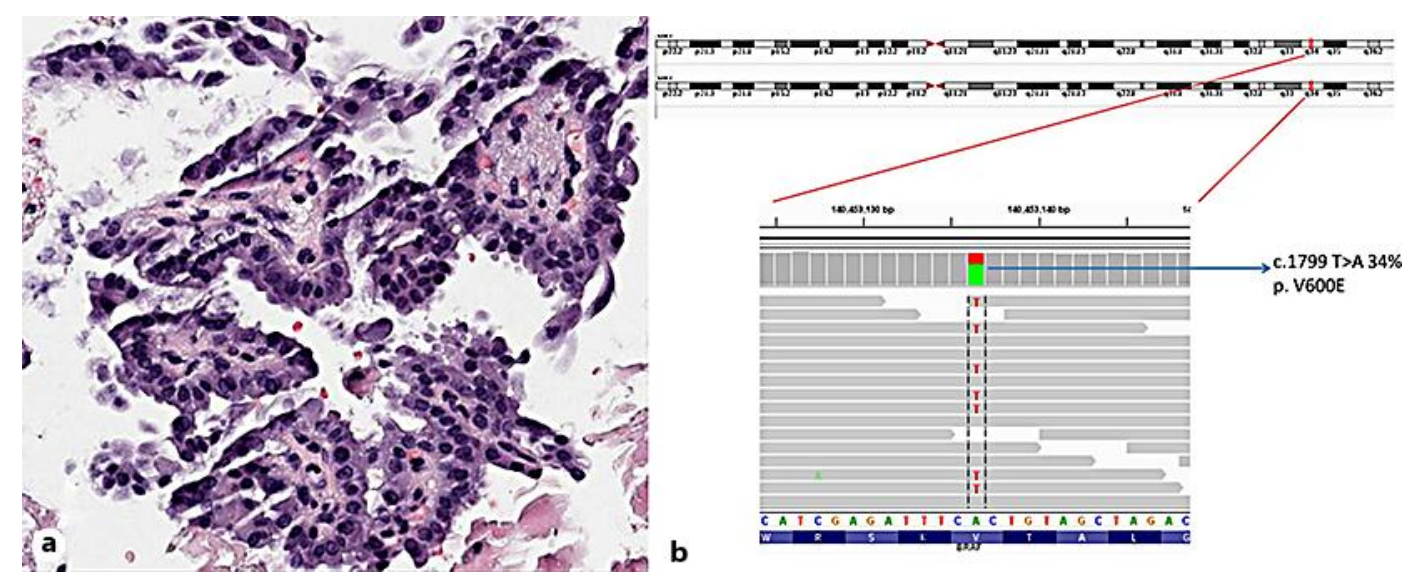

Fig. 1. a Histological section of a tumor metastatic to the lymph node, showing a papillary architecture and cytologic features of PTC $(\times 200$ magnification $)$. b Next-generation sequencing demonstrating base substitution of c.1799 $\mathrm{T}>\mathrm{A}$ in $B R A F$ at a mutant allele frequency of $34 \%$, which gives rise to $\mathrm{V} 600 \mathrm{E}$.
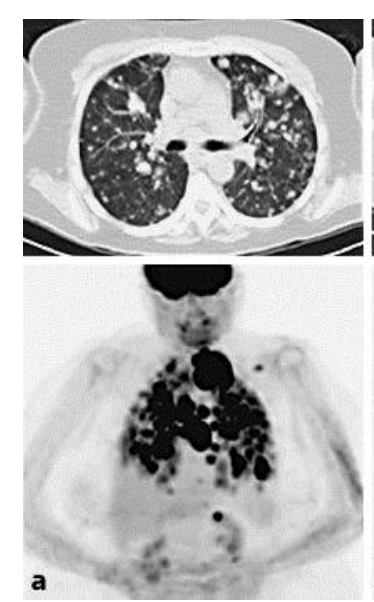
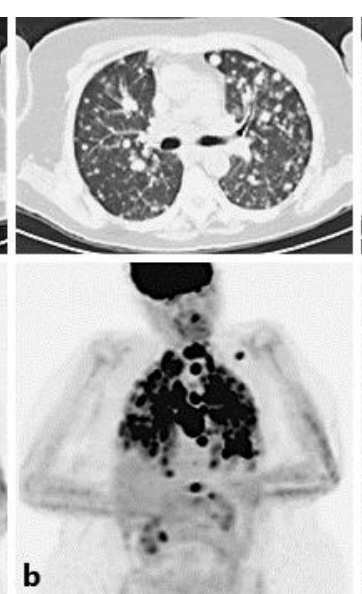
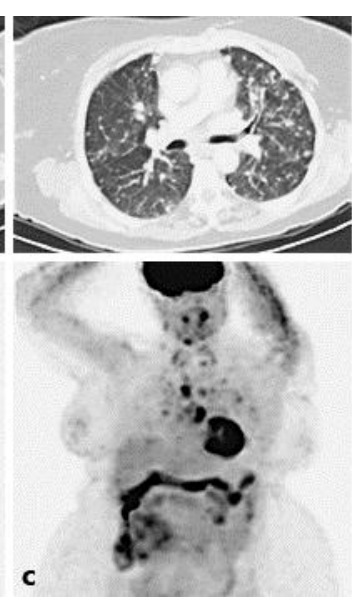

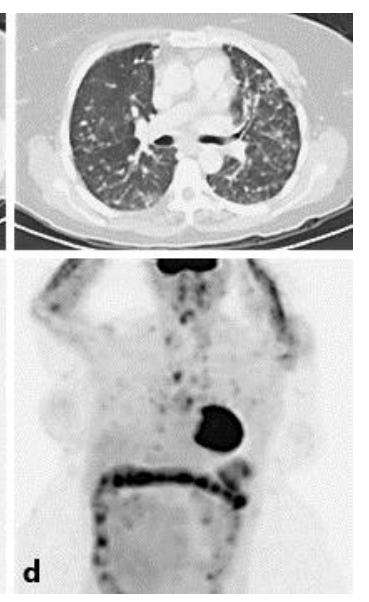

Fig. 2. Top panels are axial views of the thorax with CT imaging, and bottom panels are PET images. a Recurrent metastatic disease through the thorax. $\mathbf{b}$ After the second course of RAI treatment, to which the patient was refractory. c Two months after initiation of vemurafenib treatment. $\mathbf{d}$ Six months on vemurafenib treatment. 